\title{
AVALIAÇÃO DE DIFERENTES SANIFICANTES NA QUALIDADE MICROBIOLÓGICA DE MANDIOQUINHA-SALSA MINIMAMENTE PROCESSADA
}

\author{
Evaluation of different sanitizers on the microbiological \\ quality of fresh-cut peruvian carrot
}

\author{
Elisângela Elena Nunes ${ }^{1}$, Eduardo Valério de Barros Vilas Boas², \\ Andréa Luiza Ramos Pereira Xisto ${ }^{2}$, Suzana Chitarra Leme ${ }^{2}$, Marisa Carvalho Botelho ${ }^{2}$
}

\begin{abstract}
RESUMO
Objetivou-se, neste trabalho, avaliar a eficiência dos sanificantes hipoclorito de sódio, peróxido de hidrogênio e dicloro isocianurato de sódio aplicados antes e após o processamento mínimo de mandioquinha-salsa. Foram utilizadas raízes de mandioquinhasalsa da cultivar Amarela de Senador Amaral adquiridas no comércio local. Para limpeza superficial das raízes foram utilizados detergente e água. Após, as raízes foram divididas em dois grupos: controle (não sanificado) e sanificado, por imersão em dicloro isocianurato de sódio $100 \mathrm{mg} . \mathrm{L}^{-1}$, por 15 minutos. Em seguida foram descascadas, fatiadas em rodelas e imersas nos seguintes sanificantes: hipoclorito de sódio (25, 50 e $100 \mathrm{mg} . \mathrm{L}^{-1}$, por 10 minutos), peróxido de hidrogênio (3 e 6\%, por 5 minutos) e dicloro isocianurato de sódio (50, 100 e $200 \mathrm{mg} \cdot \mathrm{L}^{-1}$, por 10 minutos); embaladas e armazenadas em câmara fria $\left(5 \pm 1^{\circ} \mathrm{C}\right.$ e $98 \%$ UR), durante 15 dias. As análises realizadas a cada 3 dias foram $\mathrm{pH}$, acidez titulável, sólidos solúveis, colimetria e pesquisa de Salmonella sp.. As variáveis $\mathrm{pH}, \mathrm{AT}$ e SS não foram influenciadas pelos tratamentos utilizados. A presença de coliformes $45{ }^{\circ} \mathrm{C}$ e Salmonella sp. não foi detectada, durante o armazenamento de mandioquinha-salsa minimamente processada, controle ou sanificada e foram observadas contagens baixas para coliformes $35^{\circ} \mathrm{C}$. A sanificação do produto antes do processamento com dicloro isocianurato de sódio $100 \mathrm{mg} . \mathrm{L}^{-1} \mathrm{e}$ condições higiênico-sanitárias adequadas, durante o processamento, possibilita a obtenção de um produto com padrão microbiológico de acordo com a legislação de alimentos, até o $15^{\circ}$ dia de armazenamento.
\end{abstract}

Termos para indexação: Segurança alimentar, processamento mínimo, armazenamento.

\section{ABSTRACT}

The objective of this work was to evaluate the efficiency of the sanitizers sodium hypochlorite, hydrogen peroxide and sodium dichloro isocianurate applied before and after the minimum processing of the Peruvian carrot. Peruvian carrots of the cultivar Amarela de Senador Amaral purchased in the local commerce were utilized. Detergent and water were used for the surface cleaning of the roots. Afterwards, the roots were divided into two groups: control (not sanitized) and sanitized, for immersion into 100mg.L $\mathrm{L}^{-1}$ sodium dichloro isocyanurate for 15 minutes. They were then peeled, sliced into rings and immersed into the following sanitizers: sodium hypochlorite (25, 50 and $100 \mathrm{mg} . \mathrm{L}^{-1}$ for 10 minutes), hydrogen peroxide ( 3 and $6 \%$, for 5 minutes) and sodium dichloro isocyanurate (50, 100 and 200 mg. $\mathrm{L}^{-1}$ for 10 minutes); packed and stored in cold chamber $\left(5 \pm 1^{\circ} \mathrm{C}\right.$ and $\left.98 \% \mathrm{RH}\right)$ for 15 days. The following analyses were performed every 3 days: $\mathrm{pH}$, titrable acidity, soluble solids, coliforms determination and search for Salmonella. The $\mathrm{pH}$, AT and SS variables were not affected by the used treatments. The presence of coliforms at $45^{\circ} \mathrm{C}$ and Salmonella was not detected during the storage of either control or sanitized fresh-cut Peruvian carrots and low counts for coliforms at $35^{\circ} \mathrm{C}$ were found. The sanitization of the produce before processing with $100 \mathrm{mg} . \mathrm{L}^{-1}$ sodium dichloro isocyanurate and hygienic-sanitary conditions during processing allow obtaining a produce with microbiological standard according to the food legislation until the $15^{\text {th }}$ day of storage.

Index terms: Food safety, fresh-cut, storage.

(Recebido em 20 de outubro de 2008 e aprovado em 19 de agosto de 2009)

\section{INTRODUÇÃO}

Alimentos minimamente processados devem ser similares ao produto fresco, porém, com qualidade microbiológica garantida (Wiley, 1997) pela redução dos microorganismos patogênicos e deteriorantes. Isso favorece o aumento da vida útil do produto, uma vez que tais microorganismos, em concentrações elevadas, prejudicam a saúde e alteram as características sensoriais do produto (Silva et al., 2006).

Sendo assim deve-se utilizar água de boa qualidade, bem como fazer a higienização dos frutos e hortaliças e dos equipamentos e utensílios, de forma a evitar contaminação cruzada e aumentar a segurança microbiológica dos alimentos minimamente processados (Suslow, 1997; Antoniolli et al., 2005).

${ }^{1}$ Centro Universitário UNIRG - Av. Rio de Janeiro entre ruas 9 e 10, Centro - Gurupi, TO - 77435-100 - elisanunescarvalho@hotmail.com

2Universidade Federal de Lavras/UFLA - Departamento de Ciência dos Alimentos/DCA - Lavras, MG 
A escolha e a aplicação adequada do sanificante químico, em frutas e hortaliças minimamente processadas, são fundamentais para a indústria de alimentos. Esses sanificantes variam em sua habilidade de destruir microrganismos. Sua efetividade depende das características físicas e químicas do vegetal, do tipo de microrganismo alvo, do tempo de contato, da concentração e temperatura de solução. Alguns sanificantes são apropriados para o uso em lavagem direta das superfícies dos alimentos inteiros ou processados, outros somente para processos de lavagem com água em equipamentos ou recipientes e aparelhos (Sapers \& Simmons, 1998).

Encontram-se disponíveis para a sanificação um grande número de marcas comerciais de compostos à base de cloro e sanificantes alternativos (Reis et al., 2008). O cloro, nas suas várias formas, consiste no sanificante mais utilizado em alimentos. Os compostos à base de cloro são germicidas de amplo espectro de ação, que reagem com as proteínas da membrana das células microbianas, interferindo no transporte de nutrientes e promovendo a perda de componentes celulares (Vanetti, 2004). O hipoclorito de sódio $(\mathrm{NaOCl})$ corresponde ao sanificante químico de maior utilização, em função de sua rápida ação, fácil aplicação e completa dissociação em água (Antoniolli et. al., 2005). Entretanto, apresenta o inconveniente de liberar trihalometanos e cloraminas, subprodutos potencialmente carcinogênicos, quando hidrolisados (Wiley, 1994).

O dicloro isocianurato de sódio é um composto clorado orgânico comercializado na forma de pó ou comprimidos efervescentes. Por atender a um processo específico de fabricação para uso em alimentos, não libera metais pesados e trihalometanos, sendo seguro para o manuseio e consumo. Assim, a substituição dos compostos clorados inorgânicos, como o hipoclorito de sódio, pelo NaDCC, para uso em alimentos já está consolidada, desde a década de 90 nos EUA e Europa (Macedo, 2001).

$\mathrm{O}$ peróxido de hidrogênio $\left(\mathrm{H}_{2} \mathrm{O}_{2}\right)$ é classificado como um composto GRAS (Geralmente Reconhecido Como Seguro) para uso em alimentos como agente alvejante, oxidante, redutor e antimicrobiano. O principal objetivo do tratamento com peróxido de hidrogênio é estender a vida de prateleira por redução da população de microrganismos deterioradores na superfície do produto (Sapers \& Simmons, 1998). Resíduos em frutas e hortaliças tratadas com peróxido de hidrogênio podem ser eliminados passivamente pela ação de catalase endógena, ou ativamente, pelo enxágue imediatamente após o tratamento, para evitar reações entre o peróxido de hidrogênio e constituintes do alimento que poderão afetar a qualidade ou a segurança do produto (Vanetti, 2004).
A mandioquinha-salsa é uma das hortaliças que apresenta grande demanda na forma minimamente processada. Entretanto, diversos cuidados devem ser tomados no processamento mínimo, relacionados à espessura do corte, controle do metabolismo, uso de refrigeração (Machado, 2008) e qualidade microbiológica. Objetivou-se, neste trabalho, avaliar a eficiência de diferentes sanificantes na manutenção da qualidade microbiológica de mandioquinhas-salsa minimamente processadas armazenadas $\left(5^{ \pm} 1^{\circ} \mathrm{C}\right.$ e $\left.90 \pm 5 \%\right)$, por quinze dias.

\section{MATERIAL E MÉTODOS}

Mandioquinhas-salsa cultivar Amarela de Senador Amaral foram adquiridas no comércio local de Lavras MG. Inicialmente, as raízes foram selecionadas, descartando-se aquelas que apresentavam injúrias, lavadas em água corrente com detergente neutro, sanificadas em solução de dicloro isocianurato de sódio (Hidrosan $\left.{ }^{\circledR}\right) 100$ mg. $\mathrm{L}^{-1}$ por 15 min, drenado o excesso de solução sanificante com auxílio de peneiras plásticas e secas ao ar. Em seguida, foram descascadas manualmente, fatiadas $(1 \mathrm{~cm}$ de espessura) com auxílio de multiprocessador MASTER AT. As fatias foram imersas nas seguintes soluções: dicloro isocianurato de sódio $50 \mathrm{mg} . \mathrm{L}^{-1}, 100 \mathrm{mg} . \mathrm{L}^{-1} \mathrm{e} 200 \mathrm{mg} . \mathrm{L}^{-1}$ por $10 \mathrm{~min}$; hipoclorito de sódio $25 \mathrm{mg} . \mathrm{L}^{-1}, 50 \mathrm{mg} . \mathrm{L}^{-1} \mathrm{e} 100$ mg. $\mathrm{L}^{-1}$ por $10 \mathrm{~min}$; peróxido de hidrogênio $(3$ e $6 \%)$ por 5 min.; controle (sem sanificação), sendo o excesso de solução sanificante removido por escoamento através de peneiras plásticas. $\mathrm{O}$ delineamento estatístico utilizado foi blocos casualizados (DBC), sendo realizados dois blocos. Práticas de higiene foram utilizadas para desinfecção do ambiente, facas e utensílios, utilização de luvas, máscaras e touca pelos manipuladores.

As raízes processadas foram acondicionadas em embalagens rígidas de polipropileno $(15 \times 11,5 \times 4,5 \mathrm{~cm})$. As embalagens contendo cerca de $150 \mathrm{~g}$ de raízes minimamente processadas foram armazenadas em câmara fria $\left(5 \pm 1^{\circ} \mathrm{C}\right.$ e $90 \pm 5 \%$ UR), por um período de 15 dias, e as amostras retiradas para análises a cada 3 dias. As análises foram:

pH - foi medido em potenciômetro com eletrodo indicador de vidro, da marca Schott Handylab, segundo a técnica da Association of Official Analytical Chemists-AOAC (2005).

Acidez titulável (\% de ácido málico) - foi determinada por titulação com solução padronizada de $\mathrm{NaOH} 0,01 \mathrm{~N}$, tendo como indicador a fenolftaleína, de acordo com o Instituto Adolfo Lutz (1985).

Sólidos solúveis ( $\left.{ }^{\circ} \mathrm{Brix}\right)$ - foram determinados por refratometria, utilizando refratômetro digital ATAGO PR100, segundo AOAC (2005). 
Determinação de coliformes 35 e $45^{\circ} \mathrm{C}$ - foram coletadas, assepticamente, vinte e cinco gramas de amostras, os quais foram diluídos em $225 \mathrm{~mL}$ de água peptonada a $0,1 \%$ estéril e, a partir dessa, foram preparadas diluições seriadas. Os coliformes $35^{\circ} \mathrm{C}$ foram quantificados utilizando-se a técnica do número mais provável (NMP). $\mathrm{O}$ teste presuntivo foi realizado com a inoculação de alíquotas da amostra em série de três tubos, contendo tubos de Durhan e caldo lauril sulfato triptose (LST), sendo incubados a $35^{\circ} \mathrm{C}$ por 24 a $48 \mathrm{~h}$, considerando-se tubos positivos para coliformes $35^{\circ} \mathrm{C}$ aqueles que apresentavam turvação e formação de gás. Os coliformes a $45^{\circ} \mathrm{C}$ foram quantificados utilizandose a técnica do NMP. Alíquotas foram transferidas dos tubos positivos do teste presuntivo para tubos contendo caldo Escherichia coli (EC) com tubos de Durhan; os tubos foram incubados a $45^{\circ} \mathrm{C}$ por 24 a $48 \mathrm{~h}$, considerandose tubos positivos para coliformes a $45^{\circ} \mathrm{C}$ aqueles que apresentavam turvação e formação de gás. Utilizou-se metodologia descrita no Manual de Análises Microbiológicas de Alimentos (Silva et al., 2007). Os resultados foram expressos em $\log _{10} \cdot \mathrm{g}^{-1}$ de mandioquinhasalsa minimamente processada.

Pesquisa de Salmonella sp. - foi realizada em 25 g do produto minimamente processado com pré-enriquecimento, em água peptonada tamponada, com incubação a $35^{\circ} \mathrm{C}$, por $24 \mathrm{~h}$, seguida de enriquecimento seletivo, em caldo tetrationato e caldo Rappaport incubados a $35^{\circ} \mathrm{C}$, por $24 \mathrm{~h}$. $\mathrm{O}$ isolamento foi realizado em meio de cultura Ramback marca Merck (Silva et al., 2007).

As análises estatísticas foram realizadas com o auxílio do programa SISVAR (Ferreira, 2000). Após análise de variância, as médias, quando significativas, foram comparadas, pelo teste de Tukey, a $1 \%$ e $5 \%$ de probabilidade.

\section{RESULTADOS E DISCUSSÃO}

$\mathrm{O} \mathrm{pH}$, acidez titulável e sólidos solúveis não foram influenciados pelos fatores tempo de armazenamento e tipo de sanitizante isoladamente, tampouco pela interação entre eles. No presente estudo, encontrou-se um $\mathrm{pH}$ médio de 6,81, acidez titulável em média de $0,15 \%$ de ácido málico e 4,1 ${ }^{\circ}$ Brix , em média, de sólidos solúveis. A acidez, em produtos minimamente processados, é benéfica sob o ponto de vista microbiológico, pois inibe o crescimento de patógenos nocivos à saúde humana (Mattiuz et al., 2004). A baixa acidez e pH próximo da neutralidade da mandioquinha-salsa podem contribuir para a proliferação de microrganismos.

Não foram detectados coliformes $45^{\circ} \mathrm{C}$, bem como Salmonella sp. nas fatias de mandioquinha-salsa controle e sanificadas, nos 15 dias de armazenamento, demonstrando a eficácia da primeira sanificação com dicloro isocianurato de sódio $100 \mathrm{mg} . \mathrm{L}^{-1}$ (antes do processamento) e dos cuidados higiênico-sanitários durante as etapas do processamento da mandioquinhasalsa. Vilas-Boas et al. (2007), ao avaliarem a eficácia de diferentes sanificantes em abobrinhas minimamente processadas também observou que só sanificação em solução de dicloroisocianurato de sódio $100 \mathrm{mg}$.L-1 por 15 minutos antes do processamento mínimo e uso de boas práticas de processamento foram suficientes para a obtenção de um produto seguro (Vilas-Boas, 2007).

Estes resultados atendem à Legislação Brasileira, ANVISA - Resolução RDC N ${ }^{0} 12$, de 2 de janeiro de 2001, do Ministério da Saúde (Brasil, 2001), que estabelece os padrões microbiológicos sanitários para alimentos. Para produtos minimamente processados ainda não existem padrões específicos. Entretanto, esses podem ser incluídos no grupo de alimentos designados como: "frutas frescas, in natura, preparadas (descascadas ou selecionadas ou fracionadas), sanificadas, refrigeradas ou congeladas, para consumo direto". A tolerância máxima para esse tipo de produto é de $5 \times 10^{2} \mathrm{NMP} . \mathrm{g}^{-1}$ ou UFC. $\mathrm{g}^{-1}$ de coliformes a 45 ${ }^{\circ} \mathrm{C}$ e ausência de Salmonella sp. em $25 \mathrm{~g}$. Portanto, os resultados microbiológicos obtidos neste trabalho apresentaram valores inferiores aos citados na legislação, caracterizando-os como um produto seguro para o consumo alimentar. Reis et al. (2008), ao estudarem o efeito de diferentes sanificantes sobre a qualidade de morangos minimamente processados observou ausência de Salmonella sp. e coliformes em todos os tratamentos, inclusive o controle. E os autores atribuem esse fato aos cuidados higiênico-sanitários, tomados durante o processamento do produto, os quais são de fundamental importância e podem contribuir para que o mesmo apresente baixa contagem microbiana.

As análises microbiológicas das amostras analisadas apresentaram baixas contagens de microrganismos do grupo de coliformes a $35^{\circ} \mathrm{C}$, sendo observadas menores contagens desses microrganismos, nas fatias tratadas com peróxido de hidrogênio a $6 \% \mathrm{e}$ dicloro isocianurato de sódio $100 \mathrm{mg} . \mathrm{L}^{-1}$ (Tabela 1).

Embora não exista na legislação padrão para coliformes a $35^{\circ} \mathrm{C}$, de forma geral, é preconizado que alimentos contendo contagens desses microrganismos da ordem de $10^{5}-10^{6} \mathrm{NMP} . \mathrm{g}^{-1}$ ou UFC.g- ${ }^{-1}$ são impróprios para o consumo humano devido à perda do valor nutricional, alterações sensoriais, riscos de deterioração e/ou presença de patógenos (Arruda et al., 2004). Estando os valores encontrados nesse trabalho bem abaixo desses valores. 
Tabela 1 - NMP.g $\mathrm{g}^{-1}$ de coliformes a $35^{\circ} \mathrm{C}$ em mandioquinhas-salsa minimamente processadas sanificadas com diferentes sanificantes e armazenadas $\left(5 \pm 1{ }^{\circ} \mathrm{C}\right.$ e $\left.90 \pm 5 \%\right)$, por 15 dias.

\begin{tabular}{lcccccc}
\hline \multicolumn{7}{c}{ Tempo de armazenamento } \\
\hline Controle & 0 & 3 & 6 & 9 & 12 & 15 \\
Dicloro 50 & $1,1 \times 10^{3}$ & $4,6 \times 10^{2}$ & $\geq 2,4 \times 10^{3}$ & $\geq 2,4 \times 10^{3}$ & $\geq 2,4 \times 10^{3}$ & $\geq 2,4 \times 10^{3}$ \\
Dicloro 100 & $4,3 \times 10$ & $4,3 \times 10$ & $2,4 \times 10^{2}$ & $7,5 \times 10$ & $7,5 \times 10$ & $0,3 \times 10$ \\
Dicloro 200 & $4,6 \times 10^{2}$ & $1,5 \times 10^{2}$ & $4,3 \times 10$ & $\geq 2,4 \times 10^{3}$ & $\geq 2,4 \times 10^{3}$ & $9,3 \times 10$ \\
$\mathrm{H}_{2} \mathrm{O}_{2} 3 \%$ & $4,3 \times 10$ & $4,6 \times 10^{2}$ & $9,3 \times 10$ & $2,3 \times 10$ & $1,1 \times 10^{3}$ & $\geq 2,4 \times 10^{3}$ \\
$\mathrm{H}_{2} \mathrm{O}_{2} 6 \%$ & $0,4 \times 10$ & $0,9 \times 10$ & $2,3 \times 10$ & $0,7 \times 10$ & $2 \times 10$ & $2,1 \times 10$ \\
$\mathrm{Hipocl.}^{25}$ & $\geq 2,4 \times 10^{3}$ & $4,3 \times 10$ & $9,3 \times 10$ & $1,1 \times 10^{3}$ & $9,3 \times 10$ & $7,5 \times 10$ \\
Hipocl. 50 & $9,3 \times 10$ & $7,5 \times 10$ & $4,6 \times 10^{2}$ & $7,5 \times 10$ & $\geq 2,4 \times 10^{3}$ & $2,1 \times 10^{2}$ \\
Hipocl. 100 & $7,5 \times 10$ & $2,3 \times 10$ & $4,3 \times 10$ & $2,1 \times 10^{2}$ & $9,3 \times 10$ & $2,4 \times 10^{2}$ \\
\hline
\end{tabular}

Dicloro-Dicloroisocianurato de sódio (mg.L-1 $)$; Hipocl.-Hipoclorito de sódio (mg.L $\mathrm{L}^{-1}$ ); $\mathrm{H}_{2} \mathrm{O}_{2}$-Peróxido de Hidrogênio

Ausência de coliformes a $45^{\circ} \mathrm{C}$ e baixas contagens de coliformes $35^{\circ} \mathrm{C}$ também foi observada por Pinto et al. (2006) e Xisto (2007), ao avaliarem o efeito de diferentes sanificantes sobre a qualidade da melancia minimamente processada e por Antoniolli et al. (2005), ao analisarem as características microbiológicas de abacaxi minimamente processado. Pinheiro et al. (2005), ao avaliarem a qualidade microbiológica de frutos minimamente processados comercializados em Fortaleza, detectaram a presença de coliformes a $35{ }^{\circ} \mathrm{C}$ e $45{ }^{\circ} \mathrm{C}$ nas amostras de goiabas, mangas, melões e mamões minimamente processados, indicando ter ocorrido contaminação dos mesmos durante a manipulação ou o armazenamento.

\section{CONCLUSÕES}

A presença de coliformes $45^{\circ} \mathrm{C}$ e Salmonella sp. não foi detectada durante o armazenamento nas fatias de mandioquinha-salsa minimamente processadas.

A sanificação do produto antes do processamento com solução de dicloro isocianurato de sódio $100 \mathrm{mg} . \mathrm{L}^{-1}$ por 15 min e condições higiênico-sanitárias adequadas, durante o processamento, possibilitam a obtenção de um produto com padrão microbiológico de acordo com a legislação de alimentos, até o $15^{\circ}$ dia de armazenamento.

\section{REFERÊNCIASBIBLIOGRÁFICAS}

ANTONIOLLI, L.R. et al. Efeito do hipoclorito de sódio sobre a microbiota de abacaxi 'Pérola' minimamente processado. Revista Brasileira de Fruticultura, Brasília, v.27, n.1, p.157-160, abr. 2005.

ARRUDA, M.C. et al. Conservação de melão rendilhado minimamente processado sob atmosfera modificada ativa. Ciência e Tecnologia de Alimentos, Campinas, v.24, n.1, p.53-58, jan./mar. 2004.

ASSOCIATION OF OFFICIALANALYTICAL CHEMISTRY. Official methods of analysis of the Association of Official Analytical Chemistry. 18.ed. Gaithersburg, 2005.

BRASIL. Ministério da Saúde. Agência Nacional de Vigilância Sanitária. Resolução RDC nº 12, de 02 de janeiro de 2001. Aprova regulamento técnico sobre os padrões microbiológicos para alimentos. Brasília, 2001. Disponível em: $<$ http://www.anvisa.gov.br/legis/resol/1201rda.htm>. Acesso em: 20 fev. 2006.

INSTITUTO ADOLFO LUTZ. Normas analíticas, métodos químicos e físicos para análise de alimentos. 3.ed. São Paulo, 1985. v.1, 533p.

\section{MACEDO, J.A.B. Subprodutos do processo de} desinfecção de água pelo uso de derivados clorados. Juiz de Fora: [s.n.], 2001. 67p.

MACHADO, M.C.M. Processamento. In: EMPRESA BRASILEIRA DE PESQUISA AGROPECUÁRIA. Mandioquinha-salsa (Arracacia xanthorrhiza). Brasília, 2008. Disponível em: <http:// www.sistemasdeproducao.cnptia.embrapa.br/ FontesHTML/Mandioquin...aSalsa/ processamento.htm>. Acesso em: 30 maio 2009. 
MATTIUZ, B.H.; MIGUEL, A.C.A.; NACHTIGAL, J.C.; DURIGAN, J.F.; CAMARGO, U.A. Processamento mínimo de uvas de mesa sem semente. Revista Brasileira de Fruticultura, Jaboticabal, v.26, n.2, p.226224, 2004.

PINHEIRO, N.M.S. et al. Avaliação da qualidade microbiológica de frutos minimamente processados comercializados em supermercados de Fortaleza. Revista Brasileira de Fruticultura, Jaboticabal, v.27, n.1, p.153156, abr. 2005.

PINTO, D.M.; VILAS-BOAS, E.V. de B.; DAMIANI, C.; VILAS-BOAS, B.M.; RODRIGUES, L.J.; COELHO, C.C.G.M.; PICCOLI, R.H. Avaliação microbiológica de produtos minimamente processados comercializados em Lavras-MG durante a estação da primavera. In: ENCONTRO NACIONAL SOBRE PROCESSAMENTO MÍNIMO DE FRUTAS E HORTALIÇAS; SIMPÓSIO ÍBERO-AMERICANO DE VEGETAIS FRESCOS CORTADOS, 4., 2006, São Pedro. Palestras, resumos, fluxogramas e Oficinas... Piracicaba: USP/ESALQ/ CYTED, 2006. p.164.

REIS, K.C. dos et al. Efeito de diferentes sanificantes sobre a qualidade de morango cv. oso grande. Ciência e Agrotecnologia, Lavras, v.32, n.1, p.196-202, jan./fev. 2008.

SAPERS, G.M.; SIMMONS, G.F. Hydrogen peroxide disinfection of minimally processed fruits and vegetables. Food Tecnology, Chicago, v.52, n.2, p.48-52, Feb. 1998.

SILVA, E.O.; BASTOS, M.S.R.; ALVES, R.E.; SOARES, N.F.F.; PUSCHMANN, R. Segurança microbiológica em frutas e hortaliças minimamente processadas. In:
SIMPÓSIO ÍBERO-AMERICANO DE VEGETAIS FRESCOS CORTADOS, 1., 2006, São Pedro. Anais... São Pedro, 2006.

SILVA, N. da; JUNQUIRA, V.C.A.; SILVEIRA, N.F.A.; TANIWAKI, M.H.; SANTOS, R.F.S. dos; GOMES, R.A.R. Manual de métodos de análise microbiológica de alimentos. 3.ed. São Paulo: Varela, 2007. 552p.

SUSLOW, T. Postharvest chlorination: basic properties and key points for effective disinfection. In: ANNUAL WORKSHOP FRESH-CUT PRODUCTS: MAINTAINING QUALITY AND SAFETY, 5., 1999, Davis. Proceedings... Davis: University of California, 1997. Section 9c, p.8.

VANETTI, M.C.D. Segurança microbiológica em produtos minimamente processados. In: ENCONTRO NACIONAL SOBRE PROCESSAMENTO MÍNIMO DE FRUTAS E HORTALIÇAS, 3., 2004, Viçosa, MG.

Palestras, Resumos e Oficinas... Viçosa, MG: UFV, 2004. p.30-32.

VILAS-BOAS, B.M. Prolongamento da vida útil e manutenção da qualidade de abobrinha 'Menina Brasileira` minimamente processada. 2007. Tese (Doutorado em Ciência dos Alimentos)-Universidade Federal de Lavras, Lavras, 2007.

WILEY, R.C. Frutas y hortalizas mínimamente procesadas y refrigeradas. Zaragoza: Acribia, 1997.

WILEY, R.C. Minimally processed refrigerated fruits and vegetables. London: Chapman \& Hall, 1994. 357p.

XISTO, A.L.R.P. Qualidade de melancia minimamente processada. 2007. Tese (Doutorado em Ciência dos Alimentos)-Universidade Federal de Lavras, Lavras, 2007. 